\title{
Hı deficiency in the galaxy cluster ACO 3627
}

\section{ATCA $^{\star}$ observations in the Great Attractor region}

\author{
B. Vollmer ${ }^{1,2,8}$, V. Cayatte ${ }^{1}$, W. van Driel ${ }^{1,3}$, P. A. Henning ${ }^{4}$, R. C. Kraan-Korteweg ${ }^{1,5}$, C. Balkowski ${ }^{1}$, \\ P. A. Woudt ${ }^{6,7}$, and W. J. Duschl ${ }^{2,8}$
}

1 Observatoire de Paris, DAEC, UMR 8631, CNRS et Université Paris 7, 92195 Meudon Cedex, France

2 Institut für Theoretische Astrophysik der Universität Heidelberg, Tiergartenstraße 15, 69121 Heidelberg, Germany

3 Unité Scientifique Nançay, CNRS USR 704B, Observatoire de Paris, 92195 Meudon Cedex, France

4 Institute for Astrophysics, University of New Mexico, 800 Yale Boulevard, NE, Albuquerque, NM 87131, USA

5 Departamento de Astronomía, Universidad de Guanajuato, Apdo. Postal 144, Guanajuato, Gto. 36000, Mexico

6 Department of Astronomy, University of Cape Town, Rondebosch, 7700 South Africa

7 European Southern Observatory, Karl-Schwarzschild-Str. 2, 85748 Garching, Germany

8 Max-Planck-Institut für Radioastronomie, Auf dem Hügel 69, 53121 Bonn, Germany

Received 19 September 2000 / Accepted 12 January 2001

\begin{abstract}
ATCA $21 \mathrm{~cm}$ Hi observations of the rich galaxy cluster ACO 3627 in the Great Attractor region are presented. Three fields of $30^{\prime}$ diameter located within one Abell radius of ACO 3627 were observed with a resolution of $15^{\prime \prime}$ and an rms noise of $\sim 1 \mathrm{mJy} /$ beam. Only two galaxies were detected in these fields. We compare their Hi distribution to new optical $R$-band images and discuss their velocity fields. The first galaxy is a gas-rich unperturbed spiral whereas the second shows a peculiar Hi distribution. The estimated $3 \sigma$ HI mass limit of our observations is $\sim 710^{8} M_{\odot}$ for a line width of $150 \mathrm{~km} \mathrm{~s}^{-1}$. The non-detection of a considerable number of luminous spiral galaxies indicates that the spiral galaxies are Hi deficient. The low detection rate is comparable to the Hi deficient Coma cluster (Bravo-Alfaro et al. 2000). ACO 3627 is a bright X-ray cluster. We therefore suspect that ram pressure stripping is responsible for the Hi deficiency of the bright cluster spirals.
\end{abstract}

Key words. galaxies: interactions - galaxies: ISM - galaxies: kinematics and dynamics

\section{Introduction}

Spiral galaxies in clusters are Hi deficient compared to similar galaxies (equivalent size and morphological type) in the field (see, e.g., Chamaraux et al. 1980). In the Virgo cluster, the Hi gas is stripped in the outer regions of spiral galaxies, an effect which is more pronounced for central spirals (Cayatte et al. 1990). The same tendency was observed for galaxies in the Coma cluster (Bravo-Alfaro et al. 2000). Hi deficiency seems correlated with the X-ray luminosity of clusters (Giovanelli \& Haynes 1985; see however Solanes et al. 2000).

In this context, the cluster ACO 3627 provides an excellent probe for further studies of environmental effects. ACO 3627 has recently been identified as a nearby

Send offprint requests to: B. Vollmer, e-mail: bvollmer@mpifr-bonn.mpg.de

* The Australia Telescope Compact Array (ATCA) is operated by the Australia Telescope National Facility, CSIRO, as a National Research Facility. massive galaxy cluster behind the Milky Way (KraanKorteweg et al. 1996). Its position in velocity space of $(l, b,<v>)=\left(325.3^{\circ},-7.2^{\circ}, 4844 \mathrm{~km} \mathrm{~s}^{-1}\right)-$ only $\sim 9^{\circ}$ from the predicted center of the Great Attractor (Kolatt et al. 1995) - makes ACO 3627 the most massive known cluster in the Great Attractor region. It is comparable in cluster mass and richness to the Coma cluster (Woudt 1998) but located one and a half times closer to us.

ACO 3627 also has an X-ray luminosity comparable to that of the Coma cluster (Böhringer et al. 1996). Its $\mathrm{X}$-ray distribution is not spherically symmetric, but shows indications of an ongoing cluster merger (Böhringer et al. 1996; Tamura et al. 1998). We therefore expect that its spiral galaxies might be HI deficient - similar to the spirals of the Coma cluster. This suspicion and the existence of a fairly large number of spiral galaxies make this cluster an ideal candidate for Hi deficiency studies of its cluster population.

The proximity of ACO 3627 to the Galactic plane impedes a complete and unbiased study of ACO 3627 in 
Table 1. Observations - ATCA configurations

\begin{tabular}{lll}
\hline Configuration & begin & end \\
\hline 0.375 & 1996 September 25 & 1996 September 30 \\
0.75A & 1996 November 16 & 1996 November 19 \\
$1.5 \mathrm{~A}$ & 1996 October 18 & 1996 October 22 \\
6.0B & 1996 September 4 & 1996 September 7 \\
\hline
\end{tabular}

the optical (Woudt 1998). $21 \mathrm{~cm}$ observations are, however, not affected by the dust in the cluster area. Various pointed Hi observations have been obtained for the optically identified spiral galaxies in the ACO 3627 area to noise levels of typically 3-5 mJy (Kraan-Korteweg et al. 1997). In addition, the southern Zone of Avoidance is being searched systematically for galaxies in Hi with the multi-beam receiver on the $64 \mathrm{~m}$ Parkes radiotelescope (cf. Henning et al. 1999; Henning et al. 2000 for results from the shallow survey, and Kraan-Korteweg \& Juraszek 2000 for first results in the Great Attractor region from the full-sensitivity survey). As this survey is limited to Galactic latitudes of $|b|<5.25^{\circ}$ it covers, however, only a very small fraction of the ACO 3627 cluster within its Abell radius.

To complement the above described single dish observations, we obtained detailed $21 \mathrm{~cm}$ line images using the Australia Telescope Compact Array (ATCA). Three fields were observed within the Abell radius of the cluster ACO 3627, plus an additional field in the outskirts of the cluster (to a noise level of about $1 \mathrm{mJy} /$ beam) to study the effects of the cluster environment on its members in different parts of the cluster. Unfortunately, one of the strongest known extragalactic radio sources is located at the center of the cluster, i.e. the wide-angle-tail (WAT) radio galaxy B1610-608 (see Jones \& McAdam 1996 for a detailed discussion), making the analysis of the data of the central ATCA field quite difficult.

The observations and the data reduction procedures are presented in Sect. 2. The HI detections are shown and discussed in Sect. 3. In Sect. 4, we compare our detection rate with the detection rate of the Coma cluster as derived from VLA observations (Bravo-Alfaro et al. 2000). The conclusions are given in Sect. 5 .

We assume a distance of $79 h_{50}^{-1} \mathrm{Mpc}$ for all galaxies in the ACO 3627 cluster as derived from the Tully-Fisher relation by Woudt (1998).

\section{Observations and data reduction}

We observed three fields inside the Abell radius of ACO 3627 (cf. Fig. 1) and one comparison field well outside the cluster, each in 4 different ATCA configurations (Table 1).

The observing time for each field was $\sim 12 \mathrm{hr}$ per configuration. The baselines employed spanned the range from $31 \mathrm{~m}$ to $6000 \mathrm{~m}$. Details of the observations can be found in Table 2. The first column is the name of
Table 2. Observations -Fields

\begin{tabular}{lcccc}
\hline field & $\mathrm{RA}(2000)$ & $\mathrm{DEC}(2000)$ & $H P B W$ & $\begin{array}{c}\text { rms noise } \\
(\mathrm{mJy} / \text { beam })\end{array}$ \\
\hline Comp. & $16^{\mathrm{h}} 51^{\mathrm{m}} 17^{\mathrm{s}} 5-58^{\mathrm{o}} 31^{\prime} 31^{\prime \prime}$ & $10^{\prime \prime} \times 8^{\prime \prime}$ & 1.0 \\
East & $16^{\mathrm{h}} 25^{\mathrm{m}} 22^{\mathrm{s}} 0-60^{\circ} 40^{\prime} 21^{\prime \prime}$ & $15^{\prime \prime} \times 14^{\prime \prime}$ & 1.0 \\
South-East & $16^{\mathrm{h}} 18^{\mathrm{m}} 32^{\mathrm{s}} 9-61^{\mathrm{o}} 12^{\prime} 18^{\prime \prime}$ & $14^{\prime \prime} \times 12^{\prime \prime}$ & 1.2 \\
Center & $16^{\mathrm{h}} 15^{\mathrm{m}} 10^{\mathrm{s}} 9-60^{\circ} 56^{\prime} 16^{\prime \prime}$ & $15^{\prime \prime} \times 10^{\prime \prime}$ & 1.3 \\
\hline
\end{tabular}

the field followed by the coordinates of the field center, the $H P B W(\alpha \times \delta)$ of the synthesized beam, and the rms noise. The effective size of each field is the individual antenna $H P B W=30^{\prime}$. We used a bandwidth of $32 \mathrm{MHz}\left(6900 \mathrm{~km} \mathrm{~s}^{-1}\right)$, divided into 256 channels of $125 \mathrm{kHz}\left(27 \mathrm{~km} \mathrm{~s}^{-1}\right)$ each and centered on a frequency of $1.397 \mathrm{GHz}$ (corresponding to an heliocentric velocity of $5023 \mathrm{~km} \mathrm{~s}^{-1}$ ). Thus, the effective velocity coverage ranges from $1530 \mathrm{~km} \mathrm{~s}^{-1}$ to $8510 \mathrm{~km} \mathrm{~s}^{-1}$ with a spectral resolution of $\sim 30 \mathrm{~km} \mathrm{~s}^{-1}$. The primary ATCA calibrator, PKS B1934-638, was observed at least once per $12 \mathrm{~h}$ run. Each 40 min observation of a field was bracketed by short observation of a nearby secondary calibrator: PKS B1718649. Each $u v$ database for a given configuration and a given field was processed separately using the NRAO Astronomical Image Processing System (AIPS) package. The subtraction of the continuum was found to be the main difficulty for the cluster fields due to the presence of the very strong [55 Jy] continuum source, B1610-608. This will therefore be discussed in detail.

(i) Comparison field: As there is a point source with a flux density of $\sim 180$ mJy near the field center, we CLEANed and subtracted the CLEAN components directly in the $u v$ plane (AIPS task: UVSUB). The remaining continuum was then removed by linear $u v$ interpolation with respect to frequency (AIPS task: UVLIN).

(ii) Eastern field: We made a CLEANed image of the WAT galaxy, B1610-608 shifting the phase center to coincide with the center of this galaxy and subtracted the CLEAN components directly in the $u v$ plane. In a second step another three fainter point sources were subtracted in the way described in (i). The remaining continuum was then removed by a linear $u v$ interpolation with respect to frequency.

(iii) South-eastern field: As B1610-608 lies just beyond the upper western edge of the image, its sidelobes dominate the image. We reconstructed this very extended $\left(\sim 14^{\prime}\right)$ source shifting the phase center again to coincide with the galaxy center and subtracting the CLEAN components directly in the $u v$ plane. Two point sources were then removed using the technique described in (i). As the sidelobes of B1610-608 then still dominated the image we repeated the procedure removing the WAT galaxy. At the end two linear $u v$ interpolation procedure were applied, the first with a shift on B1610-608, the second with a zero shift. 


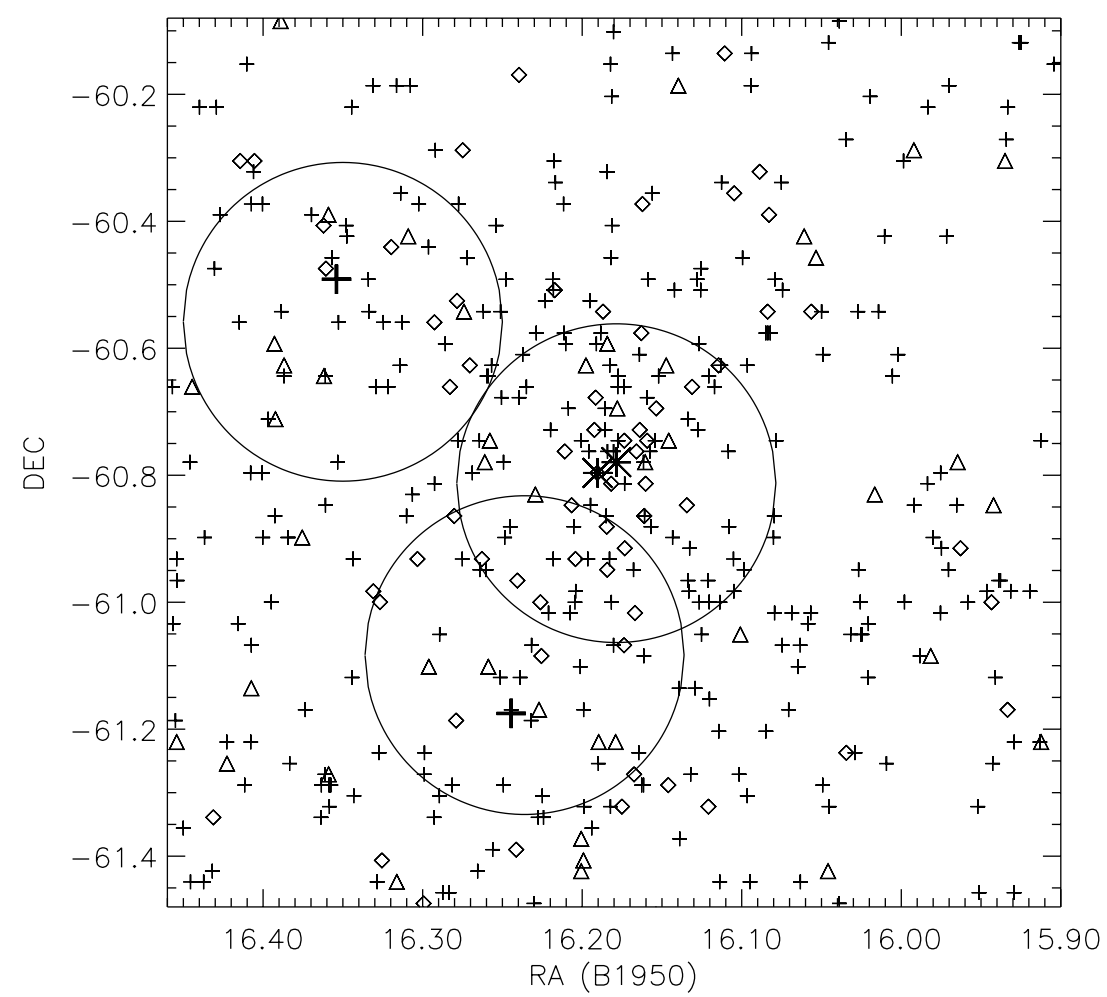

Fig. 1. Overview of the three inner observed ATCA fields. The central $\mathrm{cD}$ galaxies are marked as stars in the middle of the map. Crosses indicate spiral galaxies, diamonds ellipticals or lenticulars, and triangles unclassified galaxies. The observed fields are outlined by circles. The locations of the two detected spiral galaxies are marked as large, bold crosses

(iv) Central field: B1610-608 is located in the center of this field. We reconstructed this source and subtracted the CLEAN components directly in the $u v$ plane. The CLEANed flux is $57 \mathrm{Jy}$ for configuration 0.375 and still $55 \mathrm{Jy}$ for configuration $0.75 \mathrm{~A}$. The main problem in this field is that the frequency baselines are not flat and even show some discontinuities. The subtraction of a polynomial fit of order 4 in the image cube (AIPS task: IMLIN) resulted in an acceptable rms noise which is only a somewhat higher $(\sim 30 \%)$ than those attained in the other fields.

After combining the databases of the four configurations for each field, images were obtained using the AIPS task IMAGR applying different weightings. In the case of uniform weighting this resulted in a final resolution of $15^{\prime \prime}$ and $\mathrm{rms}$ noises of $\sim 1 \mathrm{mJy} /$ beam. As expected, the rms noise in the different fields increases with decreasing distance to B1610-608.

\section{Results}

In order to detect possible galaxy candidates, we applied two different strategies.

Since the optical galaxy diameters are known to range from $20^{\prime \prime}$ to $100^{\prime \prime}$, we analysed our data at different resolutions. We used the full resolution data as a search for an optimized study of small size galaxies. For an optimized study of larger objects $\left(\geq 30^{\prime \prime}\right)$ we degraded the resolution to about $30^{\prime \prime}$, applying a $u v$ taper of $10 \mathrm{k} \lambda$ and a weighting close to a natural weighting, resulting in beamsizes of about $30^{\prime \prime} \times 30^{\prime \prime}$. This increases the sensitivity to extended sources with diameters greater than $15^{\prime \prime} \simeq 5 \mathrm{kpc}$ by factors up to 4 compared with uniform weighting. The main galaxy search was made in a data cube with a degraded resolution of $\sim 30^{\prime \prime}$. First, for each of the four fields we added the emission of all velocity channels at this resolution above a threshold of $3 \sigma$. We then made spectra for all peaks in the integrated HI maps exceeding the $3 \sigma$ level.

In order to double check the results of these two search techniques we made small sized data cubes $\left(2^{\prime} \times 2^{\prime} \times\right.$ $250 \mathrm{~km} \mathrm{~s}^{-1}$ ) centered on the position and velocity of all Woudt (1998) galaxies in the fields. The relatively small size of the cubes allow a simple, reliable continuum subtraction. We found no additional detections using this method.

Woudt (1998) identified $\sim 80$ spiral galaxies in the three fields, out of which 20 have extinction-corrected blue luminosities $B_{\mathrm{J}}^{0}<15$ mag. In total, we found two galaxies, one in eastern field and one in south-eastern field. Their properties are listed in Table 3. The columns are: (1) galaxy name (2) RA(2000) of the optical center (3) DEC (2000) of the optical center (4) diameter of the major axis (5) diameter of the minor axis (6) extinction-corrected diameter of the major axis (7) extinction-corrected apparent $B$ magnitude (the extinctions were taken from the Schlegel et al. 1998 maps) (8) range of morphological type (9) optical heliocentric velocity (10) central HI velocity 
Table 3. Detected spiral galaxies

\begin{tabular}{|c|c|c|c|c|c|c|c|c|c|c|c|c|}
\hline Name & $\mathrm{RA}(2000)$ & $\operatorname{DEC}(2000)$ & $\begin{array}{l}D \\
\left({ }^{\prime \prime}\right) \\
(4)\end{array}$ & $\begin{array}{c}d \\
\left({ }^{\prime \prime}\right) \\
(5)\end{array}$ & $\begin{array}{l}D_{0} \\
\left({ }^{\prime \prime}\right) \\
(6)\end{array}$ & $\begin{array}{c}B_{\mathrm{J}}^{0} \\
(\mathrm{mag}) \\
(7)\end{array}$ & type & $\begin{array}{c}v_{\mathrm{opt}} \\
\left(\mathrm{km} \mathrm{s}^{-1}\right) \\
(9)\end{array}$ & $\begin{array}{c}v_{\mathrm{HI}} \\
\left(\mathrm{km} \mathrm{s}^{-1}\right) \\
(10)\end{array}$ & $\begin{array}{c}W_{20} \\
\left(\mathrm{~km} \mathrm{~s}^{-1}\right) \\
(11)\end{array}$ & $\begin{array}{c}M_{\mathrm{HI}} \\
\left(10^{9} M_{\odot}\right) \\
(12)\end{array}$ & $\begin{array}{c}M_{\mathrm{HI}} / L_{B} \\
\left(M_{\odot} / L_{\odot, B}\right) \\
(13)\end{array}$ \\
\hline WKK6801 & 162536.2 & -603619 & 38 & 16 & 47 & 15.7 & $\mathrm{Sdm}-\mathrm{Im}$ & $3518 \pm 7$ & $3529 \pm 30$ & 160 & 13.1 & 2.43 \\
\hline WKK6489 & $\begin{array}{lll}16 & 19 & 03.7\end{array}$ & -611747 & 48 & 13 & 61 & 15.1 & $\mathrm{Sb}-\mathrm{Sd}$ & $3794 \pm 58$ & $3880 \pm 30$ & 140 & 1.6 & 0.17 \\
\hline
\end{tabular}

(11) HI linewidth at 20\% (12) HI mass (13) $M_{\mathrm{HI}} / L_{B}$. For further analysis we made images of the best possible spatial resolution $\left(15^{\prime \prime}\right)$ for the detected galaxies. For the determination of integrated spectra and the total flux we use the $30^{\prime \prime}$ resolution cubes which are more sensitive for these extended sources. We will now discuss the detected galaxies in detail.

\subsection{WKK 6801}

This HI detection in the field East coincides with the optical identification WKK 6801 (see Fig. 4a). It is located at a projected distance of $2.6^{\circ}=3.5 \mathrm{Mpc}$ from the cluster center. This spiral galaxy has an extinction-corrected $B$ magnitude of $B_{\mathrm{J}}^{0}=15.7$, an extinction-corrected diameter of $D_{0}=47^{\prime \prime}$, and a radial velocity of $3519 \pm 7 \mathrm{~km} \mathrm{~s}^{-1}$ (Woudt 1998). Figure 2 shows the full resolution $\left(15^{\prime \prime}\right)$ channel maps. The galaxy is visible over a range of 6 channels. The integrated Hi spectrum measured over a $60^{\prime \prime} \times 60^{\prime \prime}$ area centered on the optical center, using the $30^{\prime \prime}$ resolution data can be seen in Fig. 3. It does not show the usual double horn feature of inclined disk galaxies but only a single peak. The derived total line flux corrected for beam attenuation is $8.9 \mathrm{Jy} \mathrm{km} \mathrm{s}^{-1}$. This corresponds to a total HI mass of $1.310^{10} M_{\odot}$ (for a distance of $79 \mathrm{Mpc}$ ). The velocity channel separation is $27 \mathrm{~km} \mathrm{~s}^{-1}$. We measured full widths at, respectively, $20 \%$ and $50 \%$ of the maximum profile height of $W_{20}=160 \mathrm{~km} \mathrm{~s}^{-1}$ and $W_{50}=110 \mathrm{~km} \mathrm{~s}^{-1}$. The error on the width is of the order of the channel separation. Hi spectra of extreme late type galaxies can show this kind of profile (Matthews et al. 1998) with comparable linewidths.

The Hi distribution map can be seen in Fig. 4b. The galaxy has a symmetric Hi distribution in the outer parts of its highly inclined disk, whereas the inner part shows two asymmetric maxima with respect to the optical galaxy center. The main maximum is located in the south-west. The maximum surface density in the center is $38 M_{\odot} / \mathrm{pc}^{2}$. The profile corresponds to those of field spirals which do not show signs of perturbation in their Hi content (Cayatte et al. 1994). The derived Hi diameter at a column density of $2.310^{20} \mathrm{~cm}^{-2}$ is $D_{\mathrm{HI}}=82^{\prime \prime}=31 \mathrm{kpc}$, leading to a mean Hi surface density of $17 M_{\odot} / \mathrm{pc}^{2}$. Woudt (1998) gives an extinction-corrected optical diameter of $47^{\prime \prime}\left(E_{(B-V)}=0.199 ;\right.$ Schlegel et al. 1998). This means that the HI diameter exceeds the optical diameter by almost a factor 2 .
The velocity field is shown in Fig. 4c. Its regular form is only perturbed at the location of the south-western maximum which could be a spiral arm. The position-velocity plot along the major axis can be seen in Fig. 4d. It shows a linearly rising rotation curve which is perturbed in the galaxy centre. We fitted a rotation curve to the data excluding $\pm 30^{\circ}$ around the minor axis. The systemic velocity is $3518 \pm 7 \mathrm{~km} \mathrm{~s}^{-1}$. The kinematical center coincides within $2^{\prime \prime}$ with the optical center given by Woudt (1998). The position angle is $\mathrm{PA}=35^{\circ}$, the inclination $i \simeq 60^{\circ}$, and the maximum rotation velocity $v_{\max } \simeq 75 \mathrm{~km} \mathrm{~s}^{-1}$.

The galaxy has an extinction-corrected magnitude of $B_{\mathrm{J}}^{0} \simeq 15.7$ (Woudt 1998). This gives an absolute magnitude of $M_{B} \simeq-18.8$ leading to an Hi mass-to-light ratio $M_{\mathrm{HI}} / L_{B} \simeq 2.43 M_{\odot} / L_{\odot, B}$ (using a distance of $79 \mathrm{Mpc}$ ). This corresponds to the median value for an $\mathrm{Sd}$ or $\mathrm{Sdm}$ galaxy (Roberts \& Haynes 1994). The maximum velocity of the rotation curve of $75 \mathrm{~km} \mathrm{~s}^{-1}$ and the line profile are also representative of a very late type galaxy.

\subsection{WKK 6489}

This HI detection in the south-eastern field matches with the optical identification of WKK 6489 (Fig. 7a). This spiral galaxy has an extinction-corrected $B$ magnitude of $B_{\mathrm{J}}^{0}=15.1$, an extinction-corrected diameter of $D_{0}=61^{\prime \prime}$, and a velocity of $3794 \pm 58 \mathrm{~km} \mathrm{~s}^{-1}$ (Woudt 1998). A very weak detection is seen in co-added 2MASS near-infrared ( $J, H, K$-band) images, whose center lies only $2^{\prime \prime} \mathrm{NW}$ of Woudt's optical position. Its estimated total magnitude in $K$ is about $14.5^{-15}$, no near-infrared colors could be derived. Its apparent major axis diameter is about $20^{\prime \prime}$, and its orientation and axial ratio appear consistent with the $R$-band image. The channel maps are shown in Fig. 5. The galaxy is clearly detected in 4 channels. The HI spectrum integrated over an area of $45^{\prime \prime} \times 45^{\prime \prime}$ (Fig. 6) shows that this galaxy has a $21 \mathrm{~cm}$ line flux which is 10 times smaller than that of WKK 6801. The derived total flux is $1.07 \mathrm{Jy} \mathrm{km} \mathrm{s}^{-1}$, corresponding to a total Hi mass of $1.610^{9} M_{\odot}$.

WKK 6489 has a corrected magnitude of $B_{\mathrm{J}}^{0} \simeq 15.1$ (Woudt 1998). This corresponds to an absolute magnitude of $M_{B} \simeq-19.4 \mathrm{mag}$ leading to an HI mass-to-light ratio $M_{\mathrm{HI}} / L_{B} \simeq 0.17 M_{\odot} / L_{\odot, B}$.

The channel width is $27 \mathrm{kms}^{-1}$, the beam size used here is $30^{\prime \prime}$. The measured full widths at, respectively, 


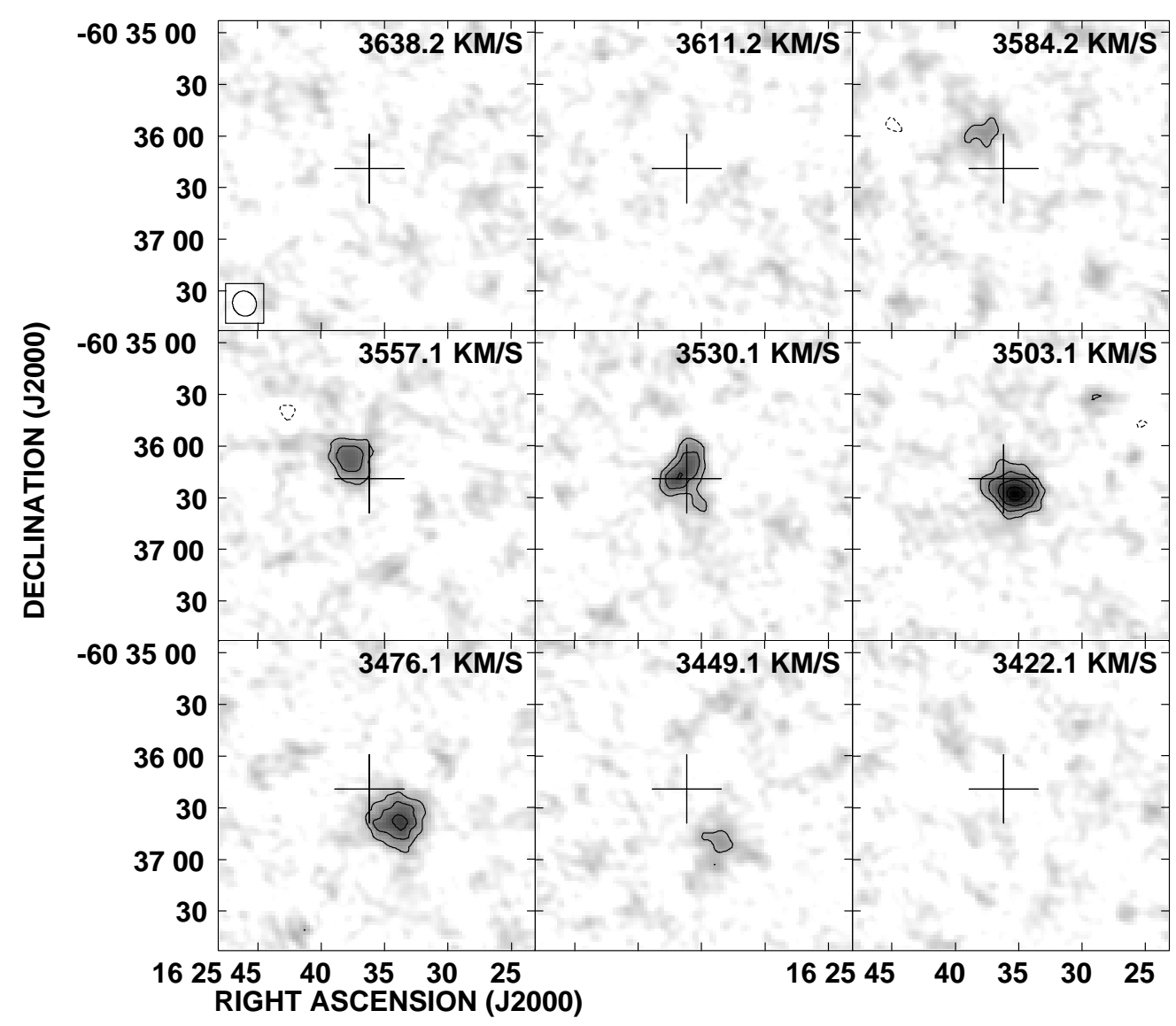

Fig. 2. Continuum subtracted channel maps of WKK 6801. The contours are $-2.79,2.79(3 \sigma), 4.65,6.50,8.36 \mathrm{mJy} / \mathrm{beam}$. The resolution is $15^{\prime \prime}$. The cross marks the optical center

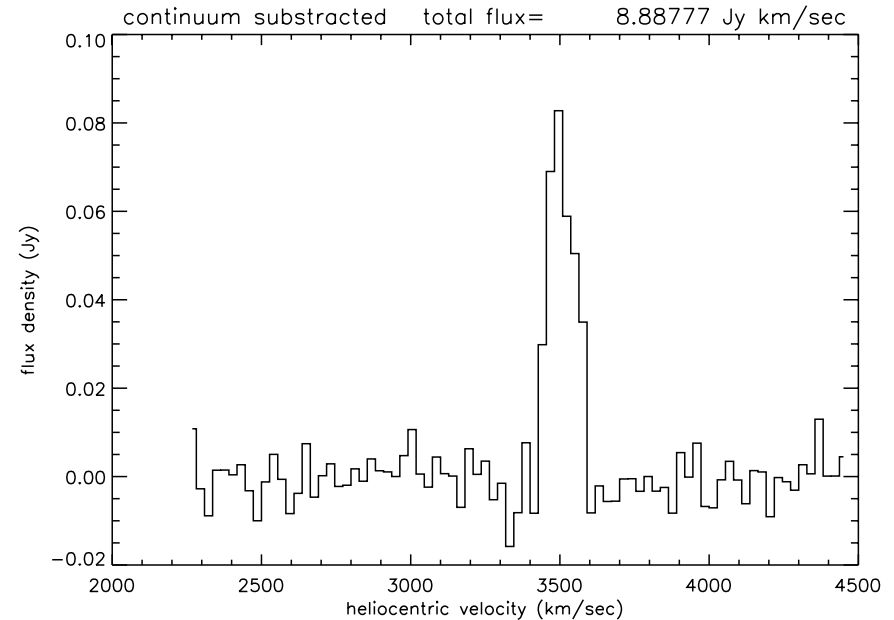

Fig. 3. The integrated spectrum (over a $60^{\prime \prime} \times 60^{\prime \prime}$ area) of WKK 6801. The channel width is $27 \mathrm{~km} \mathrm{~s}^{-1}$. The beam size is $30^{\prime \prime}$

$20 \%$ and $50 \%$ of the maximum profile height are $W_{20}=$ $140 \mathrm{~km} \mathrm{~s}^{-1}$ and $W_{50}=80 \mathrm{kms}^{-1}$. Figure $7 \mathrm{~b}$ shows the Hi brightness distribution. The cross indicates the optical center determined by Woudt (1998). The main HI emission lies south-east of the optical galaxy center. Its velocity coincides with the optical velocity within the errors. A second blob is located at a distance of $30^{\prime \prime}$ under the south-eastern end of the major axis. Woudt (1998) gives an extinction-corrected optical diameter of $61^{\prime \prime}$. This means that the south-eastern blob lies at the edge of the optical disk. The velocity field can be seen in Fig. 7c. Its peculiar behaviour could partly be due to the high noise level in the data. The position-velocity along the major axis is shown in Fig. 7d. We observe a continuous velocity field between the two distinct Hi emission blobs. Thus, the two blobs are possibly connected but our data does not permit to draw a firm conclusion. In principle the observed asymmetric Hi morphology could be due to:

- a galaxy-galaxy interaction;

- an interaction between the cluster's gravitational potential;

- ram pressure exerted by the hot intracluster medium on the fast moving galaxy (Gunn \& Gott 1972).

Since there is no optical companion, a gravitational interaction is unlikely. Moreover, its asymmetric HI gas distribution reminds that of NGC 4848 located in Coma (Bravo-Alfaro et al. 2000), a cluster which is very similar to ACO 3627 (see Sect. 4.2). N-body models simulating the gas disk of spiral galaxies falling into the Virgo cluster (Vollmer et al. 2001) show the importance of ram pressure 


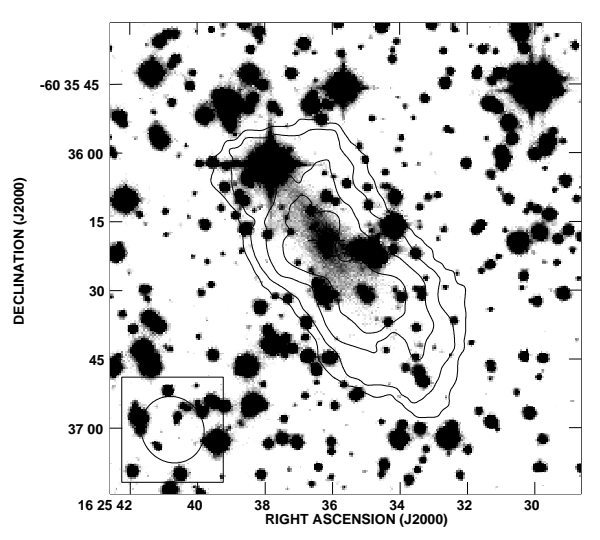

(a)

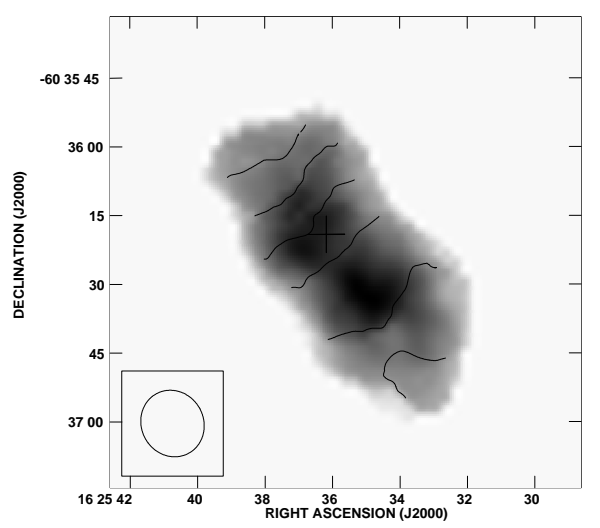

(c)

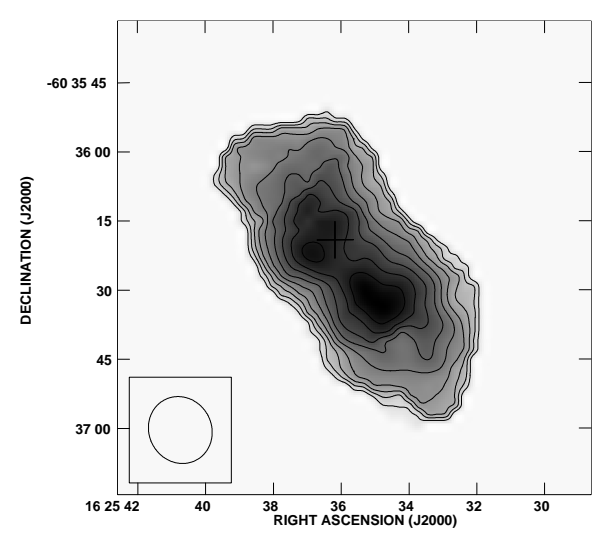

(b)

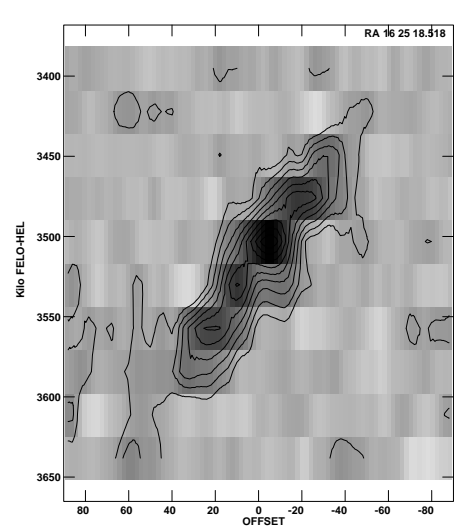

(d)

Fig. 4. Galaxy WKK 6801. a) Overlay of a high contrast optical $R$-band image and the Hi distribution with some selected contours. The Hi beam is shown in the lower left corner. b) Hi distribution. The outer contour corresponds to $2.310^{20} \mathrm{~cm}^{-2}$, the contour steps have the same value. The $15^{\prime \prime}$ beam is shown in the bottom left corner. The cross marks the optical center. c) Velocity field. The first contour in the north-east corresponds to $3560 \mathrm{~km} \mathrm{~s}^{-1}$. The contours are in steps of $-20 \mathrm{~km} \mathrm{~s}$. The cross marks the optical center. d) Position-velocity plot along the major axis. Negative position offsets correspond to the south-west direction. Contour levels are $(1,2,3,4,5,6,7,8,9) \times 0.925 \mathrm{mJy} / \mathrm{beam}$

stripping in this cluster. We expect this effect to be still more pronounced in bigger clusters as ACO 3627 or Coma. We therefore think that ram pressure is the most probable mechanism which caused the observed asymmetry.

\subsection{Number of detections}

The $3 \sigma$ detection limit in each field is $\sim 3 \mathrm{mJy} /$ beam in one velocity channel with a beamsize of $30^{\prime \prime}$. WKK 6801 and WKK 6489 represent thus $9 \sigma$ and $3 \sigma$ detections. If we assume an average $M_{\mathrm{HI}} / L_{B}=0.2 M_{\odot} / L_{\odot, B}$ (Roberts \& Haynes 1994) and a limiting Hi mass of $710^{8} M_{\odot}$ (see Sect. 4.1), we obtain a limiting luminosity $L_{B} \simeq$ $3.510^{9} L_{\odot, B}$. With a distance of $79 \mathrm{Mpc}$ this leads to $B_{\mathrm{J}}^{0}=16.2$. The number of cluster spiral galaxies with $B_{\mathrm{J}}^{0} \leq$ 16.2 within the Abell radius is 214 (Woudt 1998). The fraction between the search area (the three inner fields) and the area within an Abell radius is $\Delta \sim 0.05$. If the spiral galaxies in ACO 3627 had $M_{\mathrm{HI}} / L_{B}$ comparable to those of field galaxies, we should have detected more than 10 galaxies.

\section{Discussion}

\subsection{Detection rate}

With an HI column density sensitivity limit of $\sim 210^{20} \mathrm{~cm}^{-2}$ we are not able to detect dwarf galaxies nor low surface brightness galaxies (Pickering et al. 1997) in our fields. The galaxies we expect to be detectable will thus have linewidths of at least $W \sim 100 \mathrm{~km} \mathrm{~s}^{-1}$. We can estimate the HI mass detection limit in the following way: an assumed flat-topped spectrum of intrinsic width $W$, due to the galaxy's inclination has an observed velocity range of $\tilde{W} \simeq W\left(1-\left(\frac{d}{D}\right)^{2}\right)^{\frac{1}{2}}$, where $D$ is the optical diameter along the major axis and $d$ is the minor axis diameter. We further assume a spatial extent of one beamsize or less per velocity channel. At a distance of $79 \mathrm{Mpc}$ this implies an Hi mass limit of

$M_{\mathrm{HI}} \simeq \tilde{W} \times 310^{-3} \times 2.3610^{5} \times 79^{2} M_{\odot}$,

where $\tilde{W}$ is in $\mathrm{km} \mathrm{s}^{-1}$. Assuming $\tilde{W}=150 \mathrm{~km} \mathrm{~s}^{-1}$ gives a limit of $\sim 710^{8} M_{\odot}$, for example. 


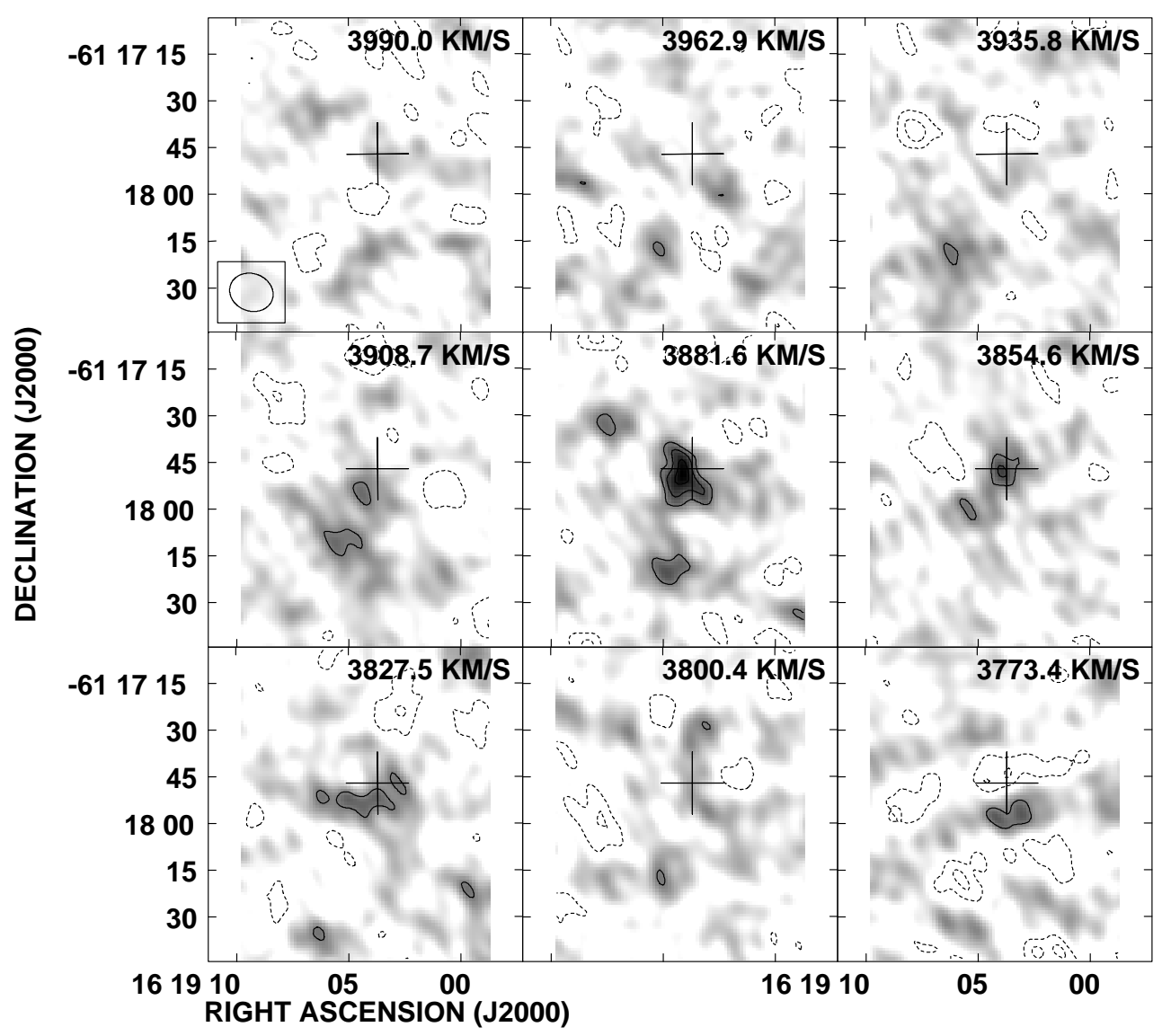

Fig. 5. The channel maps of WKK 6489 . The contours are $-2.39,-1.20,2.39(3 \sigma), 3.34,4.30 \mathrm{mJy} /$ beam. The cross marks the optical center. The resolution is $15^{\prime \prime}$

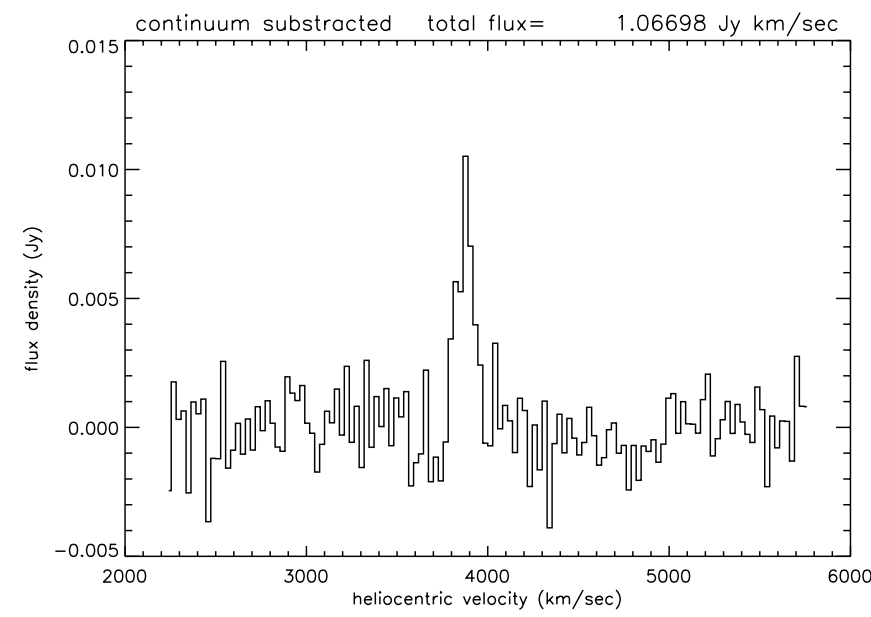

Fig. 6. The integrated spectrum of WKK 6489 over a $45^{\prime \prime} \times 45^{\prime \prime}$ area. The channel width is $27 \mathrm{~km} \mathrm{~s}^{-1}$. The beam size is $30^{\prime \prime}$

We can estimate the Hi linewidth of individual objects using the $B$-band Tully Fisher (TF) relation. Adopting the Virgo cluster $B$-band TF relation found by Yasuda et al. (1997), and using a distance of $17 \mathrm{Mpc}$ for the Virgo cluster and $79 \mathrm{Mpc}$ for ACO 3627, we obtain as TF relation for $\mathrm{ACO} 3627$ :

$B_{\mathrm{T}}^{0}=-7.92\left(\log W_{20}-2.5\right)+15.62$.
We have calculated a limiting $\mathrm{HI}$ mass-to-blue light ratio, $M_{\mathrm{HI}} / L_{\mathrm{B}}$, for each individual spiral galaxy identified in our three ACO 3627 ATCA fields by Woudt (1998), using the linewidth obtained with the TF relation and relation (1).

The high extinction makes it difficult to identify the exact morphological type of any individual object. Woudt (1998) made an attempt to classify the detected galaxies where it was possible. We decided to separate the galaxies detected and classified by Woudt (1998) into three different groups according to their morphology: group I: 22 early-type galaxies (S0/a-Sb), group II: 15 late-type galaxies (Sbc-Sd), and group III: 31 spiral galaxies without further morphological subclassification (S type). The mean limits to the mass-to-light ratio and their dipersions for these groups are

- Group I: $M_{\mathrm{HI}} / L_{B}=0.17 \pm 0.10 M_{\odot} / L_{\odot, B}$

- Group II: $M_{\mathrm{HI}} / L_{B}=0.22 \pm 0.09 M_{\odot} / L_{\odot, B}$

- Group III: $M_{\mathrm{HI}} / L_{B}=0.34 \pm 0.11 M_{\odot} / L_{\odot, B}$.

The global limit to the mass-to-light ratio for all galaxies is $M_{\mathrm{HI}} / L_{B}=0.25 \pm 0.13 M_{\odot} / L_{\odot, B}$.

For comparison, Roberts \& Haynes (1994) give the following average HI mass-to-blue light ratios and dispersions as function of morphological types: S0: $0.03 \pm$ $0.02 M_{\odot} / L_{\odot, B}, \mathrm{Sa} / \mathrm{Sb}: 0.1 \pm 0.07 M_{\odot} / L_{\odot, B}$, and $\mathrm{Sc} / \mathrm{Sd}$ : $0.3 \pm 0.15 M_{\odot} / L_{\odot, B}$. Our mean limit to the mass-to-light 


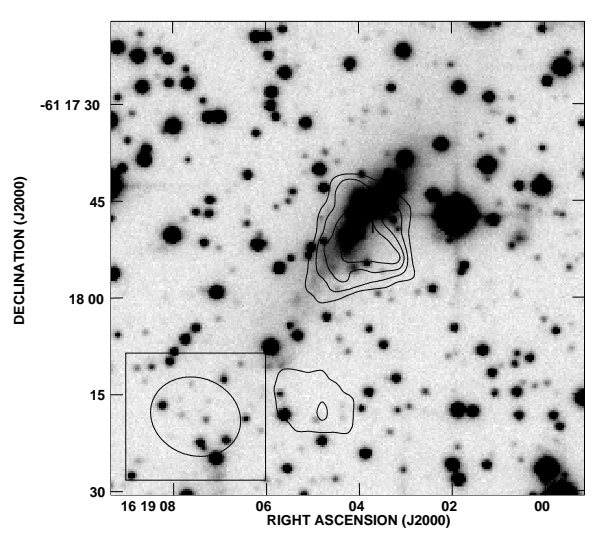

(a)

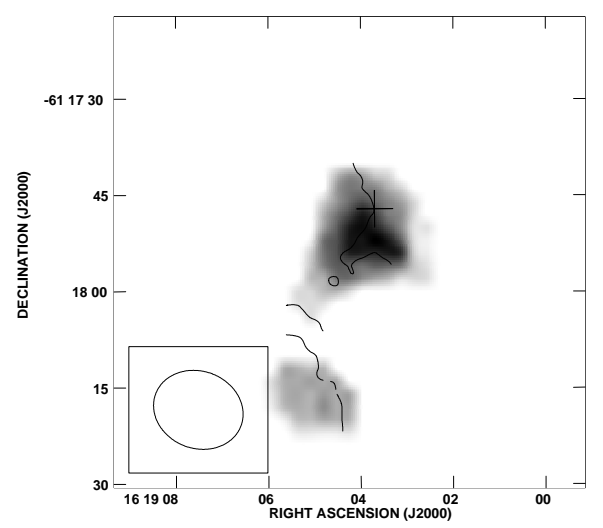

(c)

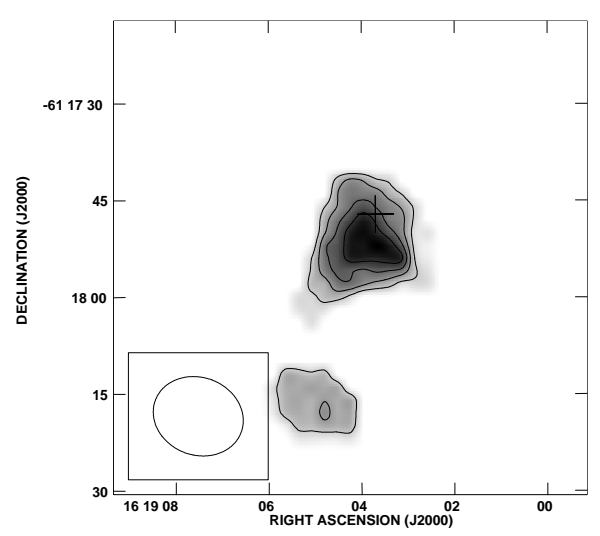

(b)

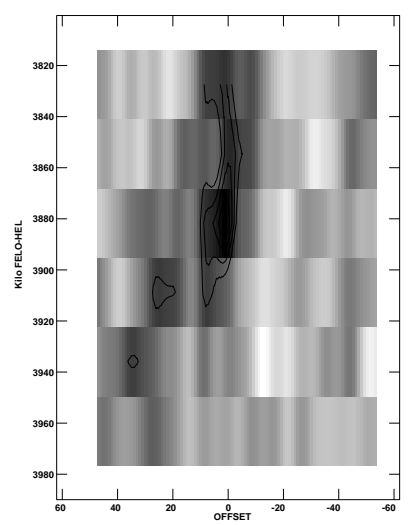

(d)

Fig. 7. WKK 6489. a) Overlay of a high contrast optical $R$-band image and the Hi distribution. b) Hi distribution. The outer contour corresponds to $1.710^{20} \mathrm{~cm}^{-2}$, the contour steps are $1.8510^{21} \mathrm{~cm}^{-2}$. The beam is shown in the bottom left corner. The cross marks the optical center. c) Velocity field. The first contour in the south-east corresponds to $3880 \mathrm{~km} \mathrm{~s}{ }^{-1}$. The next contours are 3900,3880 , and $3860 \mathrm{~km} \mathrm{~s}^{-1}$ northwards. The cross marks the optical center. The beam is shown in the upper right corner. d) Position-velocity plot along the major axis. Negative position offsets correspond to the north-west direction

ratio for the late-type (Sbc-Sd) galaxies in ACO 3627 is below the mean value given for Sc-Sd spirals by Roberts \& Haynes. Moreover, we find 8 galaxies (or 36\%) in group I (S0/a-Sb) with $M_{\mathrm{HI}} / L_{B} \leq 0.1 M_{\odot} / L_{\odot, B}$, the average for $\mathrm{Sa}-\mathrm{Sb}$ spirals found by Roberts \& Haynes, and 7 galaxies (or $47 \%$ ) in group II (Sbc-Sd) with $M_{\mathrm{HI}} / L_{B} \leq$ $0.2 M_{\odot} / L_{\odot, B}$, while 0.3 is the mean value for Sc-Sd spirals by Roberts \& Haynes. We should have detected at least some of the spirals identified in the ACO 3627 cluster, if they have $M_{\mathrm{HI}} / L_{B}$ ratios comparable to those found by Roberts \& Haynes.

In principle there are three possible explanations why they are not detected:

1. The galaxies with unknown velocities are outside the velocity range. This is, however, quite unlikely considering the velocity range covered with the ATCA observations being $\left(2300-7700 \mathrm{~km} \mathrm{~s}^{-1}\right)$ compared to the mean velocity and velocity dispersion of ACO 3627 of $<V>=4844 \mathrm{~km} \mathrm{~s}^{-1}$, respectively $\sigma_{\mathrm{V}}=848 \mathrm{~km} \mathrm{~s}^{-1}$ (cf. also Col. 9 in Table 3);
2. Some early type galaxies are misclassified as spirals. Since it is very difficult to determine morphological types due to the high extinction in the Galactic plane, misclassifications are always possible. Nevertheless, there should not be a systematic trend to misclassify lenticular galaxies with intrinsically featureless disks as spirals;

3. They are Hi deficient.

We conclude that even if we take the two former points into account there is evidence that a significant fraction of the central spiral galaxies are Hi deficient.

\subsection{Comparison with the Coma cluster detection rate}

The X-ray luminosity, velocity dispersion, galaxy magnitude distribution of ACO 3627 all are comparable to that of the Coma cluster (Woudt 1998). We will therefore compare our detection rate with that measured with the VLA in Coma by Bravo-Alfaro et al. (2000). Even if their data do not cover the whole velocity range of the cluster, they 
should not have missed a considerable number of galaxies, because their fields where centered on the galaxies with the highest $B$ luminosity $\left(B_{\mathrm{T}}^{0}<15.7\right)$. They detected 19 galaxies in a search area of $\sim 2.3 \square^{\circ}$ with an rms noise of $0.4 \mathrm{mJy} /$ beam. With our sensitivity $\left(\sim 210^{20} \mathrm{~cm}^{-2}\right)$ they would have detected 9 galaxies. This gives a detection rate of $n_{\text {Coma }}=3.9$ galaxies $/ \square^{\circ}$. We have detected 2 galaxies in a search area of $a=0.75 \square^{\circ}$ (the three central fields) leading to $n_{\mathrm{A} 3627}=2.67$ galaxies $/ \square^{\mathrm{o}}$.

In the case of spatially resolved Hi emission the Hi flux density of galaxies of the same physical size and the same total Hi mass is the same for both clusters. Therefore, we only have to correct the Coma detection rate for the search area in ACO 3627. If the HI emission is not resolved an additional correction for beam dilution effect must be taken into account. Since the galaxies in both clusters should be spatially resolved in Hi, we will consider only the first case. If we correct the Coma detection rate for the distance of ACO 3627 we get

$n_{\text {Coma }}^{\text {corr }}=n_{\text {Coma }}\left(\frac{\langle v\rangle_{\mathrm{A} 3627}}{\langle v\rangle_{\text {Coma }}}\right)^{2}=2.0$ galaxies $/ \square^{\mathrm{o}}$,

where $\langle v\rangle_{\mathrm{A} 3627}=4844 \mathrm{kms}^{-1}$ and $\langle v\rangle_{\text {Coma }}=$ $6853 \mathrm{~km} \mathrm{~s}^{-1}$. The expected number of detection for our survey is $n_{\mathrm{A} 3627}^{\mathrm{ex}}=n_{\mathrm{Coma}}^{\text {corr }} \times a \simeq 1.5$ galaxies. Thus, we have a reasonable detection rate compared to that of the Coma cluster.

Moreover, out of 21 galaxies in the three inner fields with $B_{\mathrm{T}}^{0}<15.2$ (this corresponds to the $B_{\mathrm{T}}^{0}<15.7$ in the Coma cluster) we detected two galaxies. Bravo-Alfaro et al. (2000) would have detected 9 galaxies out of 44 in the Coma cluster with our sensitivity. On the other hand, the cluster ACO 262 located at nearly the same distance as ACO 3627 and similar to the Virgo cluster, i.e. only mildly Hi deficient, has been mapped with the Westerbork array by Bravo-Alfaro et al. (1997). They detected 11 out of 25 galaxies $\left(B_{\mathrm{T}}^{0}<15.2\right)$ with a sensitivity comparable to ours. Clearly, the Hi detection rate in ACO 3627 is similar to that of the HI deficient Coma cluster and not that of A 262, indicating that the bright spiral galaxies in ACO 3627 are Hi deficient.

\section{Conclusions}

We have observed three different fields of $30^{\prime}$ diameter each within the Abell radius of the galaxy cluster ACO 3627 as well as one comparison field outside the cluster with an rms noise of $\sim 1 \mathrm{mJy} /$ beam. We have detected 2 galaxies for the first time: WKK 6801, located at a projected distance of $2.6^{\circ}=3.5 \mathrm{Mpc}$ east of the cluster center, seems to be a very late type spiral. For WKK 6489, located $1^{\circ}=1.3 \mathrm{Mpc}$ south east of the cluster center, our data does not permit to draw firm conclusions about its exact nature but it is probably an Hi deficient galaxy.

The detection rate is in reasonable agreement with that obtained for the Coma cluster (Bravo-Alfaro et al. $2000)$. There is evidence that a large fraction of spiral galaxies in the inner $1^{\circ} \times 1^{\circ}$ area are $\mathrm{HI}$ deficient. This deficiency is probably due to the interaction of the atomic gas in the galaxies with the hot intracluster gas detected in X-rays by Böhringer et al. (1996). We therefore expect that ram pressure stripping is a probable cause for the HI deficiency of the spiral galaxies in ACO 3627.

Acknowledgements. We particularly would like to thank B. Koribalski for doing one observing run and for giving important advise on the observing procedure. We also wish to thank the ATCA and ATNF staff for their kind support during the observations.

BV is supported by a TMR Programme of the European Community (Marie Curie Research Training Grant). The research of P.A.H. is supported by NSF Faculty Early Career Development (CAREER) Program award AST 95-02268.

\section{References}

Böhringer, H., Neumann, D. M., Schindler, S., \& KraanKorteweg, R. C. 1996, ApJ, 467, 168

Bravo-Alfaro, H., Szomoru, A., Cayatte, V., Balkowski, C., \& Sancisi, R. 1997, A\&AS, 126, 537

Bravo-Alfaro, H., Cayatte, V., van Gorkom, J. H., \& Balkowski, C. 2000, AJ, 119,580

Cayatte, V., van Gorkom, J. H., Balkowski, C., \& Kotanyi, C. 1990, AJ, 100, 604

Cayatte, V., Kotanyi, C., Balkowski, C., \& van Gorkom, J. H. 1994, AJ, 107, 1003

Chamaraux, P., Balkowski, C., \& Gérard, E. 1980, A\&A, 83, 38

Giovanelli, R., \& Haynes, M. P. 1985, ApJ, 292, 404

Gunn, J. E., \& Gott, J. R. 1972, ApJ, 176, 1

Henning, P. A., Staveley-Smith, L., Kraan-Korteweg, R. C., \& Sadler, E. M. 1999, PASA, 16, 35

Henning, P. A., Staveley-Smith, L., Ekers, R. D., et al. 2000, AJ, 119, 2686

Jones, P. A., \& McAdam, W. B. 1996, MNRAS, 282, 137

Kolatt, T., Dekel, A., \& Lahav, O. 1995, MNRAS, 275, 797

Kraan-Korteweg, R. C., Woudt, P. A., Cayatte, C., et al. 1996, Nature, 379, 519

Kraan-Korteweg, R. C., Woudt, P. A., \& Henning, P. A. 1997, PASA, 14, 15

Kraan-Korteweg, R. C., \& Juraszek, S. 2000, PASA, 17, 6

Matthews, L. D., van Driel, W., \& Gallagher, J. S. III 1998, AJ, 116, 1169

Pickering, T. E., Impey, C. D., van Gorkom, J. H., \& Bothun, G. D. 1997, AJ, 114, 1858

Roberts, M. S., \& Haynes, M. P. 1994, ARA\&A, 32, 115

Schlegel, D. J., Finkbeiner, D. P., \& Davis, M. 1998, ApJ, 500, 525

Solanes, J. M., García-Gómez, C., Manrique, A., et al. 2000, in Dynamics of Galaxies: from the Early Universe to the Present, ed. F. Combes, G. A. Mamon, \& V. Charmandaris, ASP Conf. Ser., 197

Tamura, T., Fukazawa, Y., Kaneda, H., et al. 1998, PASJ, 50, 195

Woudt, P. A. 1998, Ph.D. Thesis, University of Cape Town

Vollmer, B., Cayatte, V., Balkowski, C., \& Duschl, W. J. 2001, ApJ, submitted

Yasuda, N., Fukugita, M., \& Okamura, S. 1997, ApJS, 108, 417 